\title{
FATORES DE RISCO PARA DESENVOLVIMENTO DE TRANSTORNOS MENTAIS COMUNS EM ADOLESCENTES GESTANTES DO MUNICIPIO DE CERES-GO.
}

RISK FACTORS FOR DEVELOPMENT COMMON MENTAL DISORDERS IN TEEN

PREGNANCY OF CERES-GO.

\section{Suelen Marçal Nogueira}

Facer Faculdades - Unidade Ceres, Universidade Federal de Goiás, Universidade Estadual de Goiás.

suelenmnogueira@yahoo.com.br

\section{Jordana Borges Mendonça}

Faculdade de Enfermagem, Universidade Estadual de Goiás - Campus Ceres.

jordanabmendonca@gmail.com

\section{RESUMO}

Introdução: A gestação na adolescência está diretamente ligada à iniciação precoce na vida sexual. A adolescente gestante se torna vulnerável socialmente a diversos riscos, entre eles o desenvolvimento de Transtornos Mentais Comuns (TMC). Objetivo: O presente trabalho buscou identificar possíveis fatores de risco para desenvolvimento de Transtornos Mentais Comuns (TMC) e o perfil de adolescentes gestantes da cidade de Ceres-GO. Metodologia: Trata-se de uma pesquisa de campo, transversal, quantitativa, que utilizou como instrumento de coleta de dados o Self Report Questionnaire (SRQ 20) a fim de levantar os fatores de risco para desenvolvimento de TMC, e o questionário socioeconômico para levantamento de perfil das gestantes adolescentes. Resultados e Discussão: Verificou-se um número significativo de fatores de risco para TMC entre as adolescentes gestantes, onde há maior suspeição com idades entre 18 e 19 anos. As gestantes possuem baixa renda, moram de aluguel e apresentam nível de escolaridade compatível com a idade. Considerações finais: As adolescentes gestantes estão sujeitas a diversos fatores de risco que podem contribuir de forma impactante para seu adoecimento mental.

Palavras-chave: Gestantes; Adolescentes; Fatores de Risco.

\section{ABSTRACT}

Introduction: Teenage pregnancy is directly linked to early initiation into sexual life. The pregnant adolescent becomes socially vulnerable to various risks, including the development 
of Common Mental Disorders (CMD). Objective: This study aimed to identify possible risk factors for the development of Common Mental Disorders (CMD) and the profile of pregnant adolescents from the city of Ceres-GO. Methodology: This is a field research, crosssectional, quantitative, which used as a data collection instrument of the Self-Report Questionnaire (SRQ 20) to raise the risk factors for CMD development, and socioeconomic questionnaire to survey profile of pregnant adolescents. Results and Discussion: There was a significant number of risk factors for CMD among pregnant teenagers, where there is more suspicion aged 18 to 19 years. Pregnant women have low income, living rental and present educational level consistent with age. Final thoughts: The pregnant adolescents are subject to various risk factors that can contribute impactful way to his mental illness.

Key words: Pregnant; Teens; Risk Factors.

\section{INTRODUÇÃO}

A abordagem de temas relacionados à gravidez na adolescência tem salientado o caráter de problema social, partindo do pressuposto que nesta fase exista incapacidade física para gestar e incapacidade psicológica para criar, uma vez que a adolescência trata-se de uma fase cercada por transformações tanto físicas quanto psíquicas (CAMARANO, 1998).

Segundo Brandão (2003) a gravidez na adolescência é enfrentada basicamente como algo indesejável, com possíveis consequências biológicas, psicológicas e sociais negativas. $\mathrm{Na}$ circunstância de uma gestação precoce, podem ser exigidas das adolescentes atitudes psicoemocionais em que as mesmas não estejam preparadas para assumir.

Para Correia e Colaboradores (2011), a gravidez é um estágio da vida da mulher que a coloca frente a diversas alterações físicas e psíquicas associando-se a uma maior fragilidade de sua saúde mental.

Já a adolescência representa por si só uma um período frágil do desenvolvimento humano, onde ocorrem diversas transições e a busca por uma nova autenticidade de personalidade (CORREIA et al., 2011).

Figueiredo apud Correia e colaboradores (2011) relata que são diversos os fatores de risco evidenciados em gestações precoces, os que mais se destacam estão relacionados a fatores psicológicos, tais como a ausência de apoio familiar, níveis elevados de estresse, presença de sintomas depressivos, baixa expectativa com relação ao futuro.

Transtorno Mental Comum (TMC) pode ser caracterizado como transtorno que pode gerar sofrimento psíquico, comprometimento funcional e interferência na qualidade de vida 
do indivíduo que o possui. Em sua maioria trata-se de casos clínicos agudos, de remissão espontânea, sintomas somáticos, muitas vezes associados a sintomas psíquicos de natureza depressiva, ansiosa e dissociativa (ARÔCA, 2009).

Para Ludermir e Albanita apud Duarte (2010), são considerados transtornos mentais comuns os transtornos somatoformes, de ansiedade e de depressão. Com sintomas como: insônia, fadiga, irritabilidade, esquecimento, dificuldade de concentração e queixas somáticas.

Segundo Duarte (2010), a identificação de Transtornos Mentais Comuns (TMC) não significa a constatação de nenhum diagnóstico, apenas levantamentos de possíveis predisposições e fatores de risco para o adoecimento mental.

O TMC é comum entre adolescentes devido aos diversos fatores associados a este estágio da vida do ser humano, e quando está interligada a agentes predisponentes a tal tipo de transtorno, a gestação nesta fase da vida se torna um assunto de extrema relevância para a saúde pública (CORREIA et al., 2011).

Na prática da saúde torna-se clara a necessidade do apoio familiar e suporte social às adolescentes grávidas, que podem ser de grande valia no enfrentamento e diminuição dos possíveis transtornos que permeiam esta fase, fornecendo apoio, esperança e proteção às adolescentes (BAPTISTA et al., 2006).

Constitui o objetivo deste trabalho, identificar os possíveis fatores de risco para TMC em adolescentes gestantes, bem como o perfil das mesmas, levantamento essencial para o entendimento e melhoria das políticas e ações de saúde pública voltadas para esta população.

\section{METODOLOGIA}

Trata-se de um estudo transversal, quantitativo do tipo descritivo e exploratório acerca dos transtornos mentais comuns em adolescentes gestantes. Realizada nas seis Unidades de Estratégia de Saúde da Família (ESF), da cidade de Ceres-GO, sendo elas ESF Vila Pedrosa, ESF Jardim Petrópolis, ESF Jardim Sorriso, ESF Vila Nova, ESF Jardim Ribeiro e ESF São Francisco.

A população desta pesquisa inclui adolescentes gestantes, com idade entre 10 a 19 anos, vinculadas às Unidades de Estratégia de Saúde da Família (ESF) da cidade de CeresGO. Obteve-se uma amostra de 12 adolescentes gestantes que concordaram com os procedimentos, legais os sujeitos da pesquisa e assinaram o termo de consentimento, em caso de menores de idade foi solicitado assinatura de um responsável, e então foi procedida a 
coleta de dados. Foram incluídas na pesquisa adolescentes gestantes com idade entre 10 e 19 anos residentes na cidade de Ceres-GO

Foi utilizado questionário socioeconômico e o Self-Report Questionnaire (SRQ-20). O modelo de questionário Self-Report Questionnaire (SRQ-20), foi criado pela OMS com intuito de avaliar transtornos mentais comuns em países em desenvolvimento (SANTOS et al, 2009). O referido questionário é constituído por 20 questões (SRQ-20) com respostas binárias, sim ou não, respondidas conforme orientação da OMS, permitindo fazer a identificação de fatores de risco que desencadeiam possíveis Transtornos Mentais Comuns,

O projeto foi submetido ao Comitê de Ética e Pesquisa (CEP) da Pontifícia Universidade Católica de Goiás (PUC-GO) sob o ${ }^{\circ}$ de protocolo 605.711 e recebeu parecer favorável para sua execução. Em todas as fases de desenvolvimento da pesquisa foram respeitados os princípios éticos regulamentados pela Resolução no 466/2012 do Ministério da Saúde, respeitando os princípios da bioética, como autonomia, não maleficência, beneficência e justiça, e assegurando aos participantes da pesquisa privacidade e sigilo.

Os dados foram analisados e agrupados de forma quantitativa, onde para detectar se uma pessoa é considerada como caso de risco para o Transtorno Mental Comum (TMC) foi utilizado o escore de sete ou mais respostas afirmativas ( $\operatorname{sim}$ ), que valem um ponto cada uma. Este escore foi definido através da determinação da sensibilidade, especificidade e dos valores positivos e negativos.

\section{RESULTADOS E DISCUSSÃO}

Participaram desta pesquisa 12 adolescentes gestantes com uma idade média de 14,5 $(+2,87)$ anos. De acordo com dados do Instituto Brasileiro de Geografia e Estatística (IBGE) No Brasil, em 2011, 25 mil meninas entre 10 e 14 anos deram à luz, e 440 mil jovens entre 15 e 19 anos tiveram gestações não planejadas. Além disso, 21,5\% dos partos no país são realizados em mulheres com menos de 20 anos (BRASIL, 2012).

As idades gestacionais (IG) encontradas foram de 1 a 13 semanas de gestação (33\%); 14 a 26 semanas (50\%), e entre 27 a 40 semanas de gestação (17\%). Em relação à escolaridade das participantes 6 adolescentes possuem o ensino médio incompleto, representando $50 \%$ da população e as outras 6 possuem ensino médio completo, portanto o nível de escolaridade das mesmas é compatível com a idade (Tabela 1).

Segundo Melhados e colaboradores (2008), o baixo nível de escolaridade leva as adolescentes a iniciarem a vida sexual mais precocemente, este fato se relaciona em sua 
maioria à falta de suporte emocional, seja por conflitos existentes na família ou por ausência dos pais, onde as adolescentes acabam deixando de lado as expectativas escolares e profissionais.

Para Carvacho, Silva e Mello (2008) as adolescentes com idade entre 10 a 14 anos, que possuem baixo nível de escolaridade demonstram possuir menos conhecimento sobre reprodução e sexualidade, do que as adolescentes mais velhas, com idade entre 15 a 19 anos que frequentam a escola por mais tempo.

No que diz respeito à renda familiar das adolescentes, 7 (58\%) relataram possuir apenas 1 salário mínimo mensal, enquanto $3(25 \%)$ relataram a renda de 1 a 2 salários mínimos por mês e apenas $2(17 \%)$ mais de 2 salários mínimos por mês, evidenciando uma maior porcentagem de gestantes com baixa renda (Tabela 1).

Para Schuimid e Schimid (2012) os altos índices de natalidade estão relativamente mais presentes em famílias de baixa renda, enquanto os dados mostram uma diminuição da taxa de fecundidade nas classes sociais mais favorecidas. Portanto a taxa de natalidade é ascendente nas classes socioeconômico-culturais menos favorecidas, são justamente as classes que se supõe menor acesso a informações.

Das participantes $6(58 \%)$ referem estar casadas, $4(33 \%)$ amasiadas e apenas $2(8 \%)$ se dizem solteiras. Quando questionadas sobre com quem moram atualmente, $83 \%$ delas relatam morar com familiares, enquanto $17 \%$ dizem morar com outros que não sejam familiares ou colegas (Tabela 1).

Segundo o Censo de 2010, 88 mil crianças com idades entre 10 e 14 anos já estavam casadas pelo Brasil. Quando se analisa a faixa etária de 15 a 17 anos, o número de uniões dispara para 567 mil. Coordenadora da pesquisa em Belém, Maria Lúcia Chaves Lima afirmou que a gravidez é o principal motivo do casamento precoce no país, onde a união é vista como a solução para controlar a sexualidade feminina (UFJF, 2015).

O grande número de uniões nesta faixa etária demonstra que as adolescentes sofrem uma pressão social com intuito de que haja a formalização da união com seus parceiros e passem a conviver sobre o mesmo teto, mesmo sem oficializar a união ou ter independência financeira. Desta maneira bebê, mãe, e algumas vezes até mesmo o parceiro, começam a morar com seus familiares de origem, conciliando assim em um mesmo espaço vários núcleos familiares dividindo a mesma renda e a mesma organização familiar (CHALEM et al., 2007).

As uniões conjugais quando intituladas de forma precoce ou mesmo sem estrutura dos membros pode contribuir para a persistência da desvantagem social. Ao adotar e limitar-se à função de mãe e dona de casa, muitas adolescentes acabam abandonando as expectativas de 
estudo, diminuindo drasticamente suas possibilidades de escolarização e formação profissional, resultando na ineficiência de seu potencial produtivo (SOUSA; GOMES, 2009).

Tabela 1. Perfil socioeconômico das gestantes adolescentes assistidas pelas ESFs de Ceres.

\begin{tabular}{lll}
\hline Perfil Socioeconômico & n & $\%$ \\
\hline
\end{tabular}

\section{Idade (anos)}

$\begin{array}{ccc}10-13 & 1 & 8 \\ 14-17 & 4 & 33 \\ 18-19 & 7 & 58\end{array}$

IG (semanas)

$1-13 \quad 4 \quad 33$

$\begin{array}{lll}14-26 & 6 & 50\end{array}$

$27-40 \quad 2 \quad 17$

Escolaridade (anos)

Ensino médio incompleto $\quad 6 \quad 50$

Ensino médio completo ou mais $\quad 6 \quad 50$

\section{Renda Individual}

$\begin{array}{lll}1 \text { salário mínimo } & 7 & 58\end{array}$

1 - 2 salários mínimos $\quad 1 \quad 25$

<2 salários mínimos $\quad 3 \quad 17$

\section{Estado civil}

$\begin{array}{lll}\text { Casado } & 6 & 58\end{array}$

$\begin{array}{lll}\text { Solteiro } & 2 & 8\end{array}$

\begin{tabular}{lcc} 
Amasiado & 4 & 3 \\
\hline Total & 12 & 100 \\
\hline
\end{tabular}

A questão do planejamento familiar, segundo Costa (2004) deve atingir eixos de participação entre as diversas esferas que cercam o adolescente, como pais, educadores, profissionais de saúde capacitados, e ainda o próprio eu do adolescente que redige seus 
valores, crenças e atitudes que influenciam no seu comportamento sexual, estimulados pela educação formal e pelo autoconhecimento das jovens.

Caputo (2008) ressalta alguns fatores que podem ser influenciadores da gestação precoce referentes à família da adolescente, como casos de gravidez da mãe na adolescência; casos de adoção, e ainda o uso de drogas ilícitas por familiares residentes no domicílio em que a adolescente reside. Dessa forma percebe-se a importância do apoio e estrutura familiar no cotidiano da adolescente.

Muitas adolescentes, mesmo após ter o bebê continuam morando com seus pais e recebem deles apoio emocional e financeiro contribuindo assim para a minimização dos efeitos negativos desta gestação indesejada (VIEIRA et al., 2007).

Outro fator importante na ocorrência da gestação adolescente é o abandono do parceiro. Neste trabalho $58 \%$ das adolescentes entrevistadas se disseram casadas. As que se encontram solteiras contam com pouco apoio social. Com isso as gestantes adolescentes e seus filhos apresentam propensão a diversos fatores de risco, biológicos ou sociais (SILVA et al., 2009).

$\mathrm{Na}$ avaliação dos transtornos mentais comuns utilizando o questionário SRQ 20, é imprescindível salientar que para ser considerado como possível caso ou suspeição de transtorno mental comum, o entrevistado deve ter respondido sete ou mais respostas afirmativas. Só terá valor para a avaliação da saúde mental da gestante adolescente a análise geral do instrumento de pesquisa, uma vez que respostas isoladas não são consideradas no que diz respeito à identificação dos possíveis fatores de risco para o desenvolvimento de TMC.

No presente estudo 8 das adolescentes gestantes (67\%) apresentaram fatores de risco e suspeição para o desenvolvimento de TMC e apenas 4 (33\%) não apresentaram fatores de risco para o desenvolvimento de TMC. Portanto, 8 responderam sete ou mais respostas afirmativas no instrumento SRQ 20 (Figura 1).

Das adolescentes que apresentaram suspeição para o desenvolvimento de TMC, 63\% possuem idade entre 18 e 19 anos, estando com 27 a 40 semanas de gestação e $50 \%$ possuem ensino médio completo e renda de apenas 1 salário mínimo por mês.

Estudo realizado na cidade de Porto Alegre numa amostra de gestantes de média e baixa renda aponta para uma prevalência de depressão em 19,6\%, e estado de ansiedade de $59,5 \%$, destacando a escolaridade e estado civil como principais fatores associados ao TMC durante a gestação. Destaca a maior renda como fator protetor de ansiedade e a menor renda com desencadeador de depressão, uma vez que as gestantes com baixa renda apresentaram 
maio prevalência de TMC com 20,2\% das entrevistadas com tendência a sintomas depressivos e $69 \%$ a sintomas ansiosos (ALMEIDA, 2010).

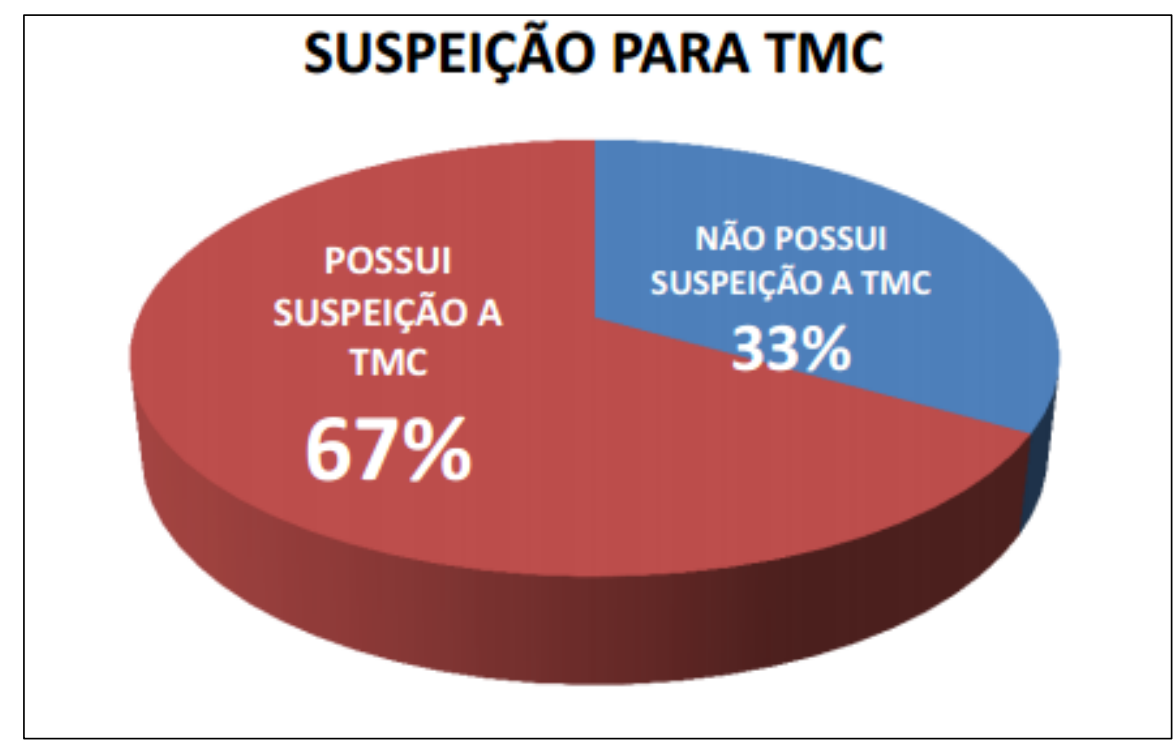

Figura 1. Relação de suspeição para o desenvolvimento de Transtornos Mentais Comuns em adolescentes gestantes assistidas pela Estratégia Saúde da Família de Ceres-GO.

Um estudo realizado na cidade de São Paulo com gestantes adolescentes evidenciou 24,3\% de possíveis casos de transtornos mentais comuns associados à baixa escolaridade (FERRI et al., 2009).

Segundo Ryan et al (2005) os principais fatores de risco para depressão durante a gestação podem ser adolescência, gravidez não planejada, sentimentos negativos sobre a gestação, mães solteiras, grande número de filhos, suporte social limitado, conflito conjugal, baixos níveis de escolaridade e renda.

Para Sabroza e colaboradores (2004) as dificuldades geradas pela gestação, em conjunto com o processo de desenvolvimento do adolescente podem ser fatores predisponentes a não aceitação das responsabilidades psicológicas, sociais e econômicas que a maternidade abrange, fazendo com que esta fase seja de difícil experiência emocional para a jovem.

Os mesmos autores afirmam que a inconstância das relações com seus parceiros durante a fase da adolescência pode também influenciar no acometimento de prejuízos psíquicos e emocionais na jovem grávida, podendo ainda ser agravados por um ambiente familiar pouco acolhedor, principalmente referente à notícia da gestação. 
Sendo assim, segundo McDonell, Limber e Connor-Godbey (2007), uma gestação adolescente que se estende em um ambiente contextualizado pelas dificuldades sociais e econômicas pode refletir em déficits das habilidades necessárias para lidar com as relações e tomadas de decisões, podendo favorecer gestações reincidentes. $O$ suporte familiar no contexto da gestação precoce tem um efeito bastante relevante diante dos estressores pessoais que acometem a adolescente.

Entende-se que no decorrer da gestação a família assume função importante no suporte da jovem tanto no meio intrafamiliar como no meio social. A interrupção ou fragilidade desse suporte, familiar e social, muitas vezes se associa ao adoecimento mental dessas adolescentes (BAPTISTA; SOUZA; ALVES, 2008).

\section{CONSIDERAÇÕES FINAIS}

Verificou-se, que adolescentes gestantes assistidas pela Estratégia da Saúde da Família da cidade de Ceres-Go, apresentam fatores de risco para desenvolvimento de Transtornos Mentais Comuns (TMC), portanto se encontram sujeitas à vulnerabilidade tanto no âmbito biológico quanto psíquico e social, que podem contribuir de forma impactante para seu adoecimento mental.

Faz-se necessário a implantação das políticas de prevenção na esfera da saúde voltadas para a questão da gestação precoce, que podem contribuir para a diminuição das estatísticas de ocorrência de gestação nesse grupo etário, fortalecendo as perspectivas e consequentemente evitando o adoecimento mental com resultados ameaçadores para o futuro das adolescentes.

\section{REFERÊNCIAS}

ALMEIDA, A. S. Transtornos Mentais em uma Amostra de Gestantes da Rede de Atenção Básica de Saúde do Sul do Brasil. Mestrado (Dissertação). Universidade Federal do Rio Grande do Sul, Porto Alegra, 2010.

ARÔCA, S.R.S. Qualidade de Vida: Comparação Entre o Impacto de Ter Transtorno Mental Comum e a Representação do Sofrimento dos Nervos em Mulheres [dissertacion]. Rio de Janeiro: Escola Nacional de Saúde Pública Sérgio Arouca/ENSP; 2009.

BAPTISTA, M. N.; BAPTISTA, A.S.D.; TORRES, E.C.R. Associação Entre Suporte Social, Depressão e Ansiedade em Gestantes. Psic [online]. 7(1), 39-48. 2006. 
BAPTISTA, M. N., SOUZA, M. S., ALVES, G. A. S. Validade entre EDEP e IPSF. PsicoUSF, 13(2), 211-220. 2008.

BRANDÃO, E. R. Individualização e Vínculo Familiar em Camadas Médias: Um Olhar Através da Gravidez na Adolescência. Tese de Doutorado, Rio de Janeiro: Instituto de Medicina Social, Universidade do Estado do Rio de Janeiro. 2003.

BRASIL. Instituto Brasileiro de Geografia e Estatística (IBGE). Censo 2012. Disponível em: >http://comuniverso.wordpress.com/2012/06/26/ibge-divulga-indices-sobre-gravideznaadolescencia/> Acesso em: 20 ago. 2014.

CAMARANO, A. A., Fecundidade e anticoncepção da população de 15-19 anos. In: Seminário Gravidez na Adolescência, Anais..., p. 35-46, Rio de Janeiro: Ministério da Saúde/Family Health International/Associação Saúde. 1998.

CAPUTO V. G., BORDIN I. A. Gravidez na adolescência e uso freqüente de álcool e drogas no contexto familiar. Rev Saúde Pública.42(3):402-10. 2008.

CARVACHO I. E, SILVA J. L. P., MELLO M. B. Conhecimento de adolescentes grávidas sobre anatomia e fisiologia da reprodução. Rev. Assoc. Med. Bras.54(1):29-35. 2008

CAVASIN S. Dossiês Adolescentes: Saúde Sexual e Reprodutiva. Disponível em: $<$ http://www.redesaude.org.br/Homepage/Dossi\%EAs/Dossi\%EA\%20Adolescentes\%2 0Sa\%FAde \%20Sexual\%20e\%20Reprodutiva\%201.pdf.> Acesso em: 23 out. 2014.

CHALEM E., MITSUHIRO S.S., FERRI C. P., BARROS M. C. M., GUINSBURG R, LARANJEIRA R. Gravidez na adolescência: perfil sócio-demográfico e comportamental de uma população da periferia de São Paulo, Brasil. Cad. Saúde Pública.23(1):177-86. 2007

CORREIA D.S., SANTOS L.V.A., CALHEIROS A.M.N., VIEIRA M.J. Adolescentes Grávidas: Sinais, Sintomas, Intercorrências e Presença de Estresse. Rev Gaúcha Enferm., Porto Alegre (RS). 32(1):40-7, mar. 2011.

COSTA A. M. Atenção integral à saúde das mulheres: Quo Vadis? Uma avaliação da integralidade na atenção à saúde das mulheres no Brasil [tese]. Brasília: Faculdade de Ciências da Saúde da Universidade de Brasília; 2004. 
DUARTE, G.J. Transtornos mentais comuns em trabalhadores rurais no corte da canade-açúcar. Mestrado (Dissertação de mestrado em Ciências Ambientais e Saúde) - Pontifícia Universidade Católica de Goiás, Goiânia, 2010.

FERRI, C. P; et al. The Impact of Maternal Experience of Violence and Common Mental Disorders on Neonatal Outcomes: a Survey of Adolescent Mothers in São Paulo, Brazil. BMC Public Health, 7, 2009.

MCDONELl, J. R; LIMBER, S. P; CONNOR-GODBEY, J. Pathways Teen Mother Support Project: Longitudinal Findings. Childre and Youth Services, Review, 840- 855. 2007.

MELHADOS, A; CARVALHO, M. J; SANT'ANA; BASTOS, M. L.; PASSARELLI; COATES, V. Adolescência e Saúde. v. 5, n. 2, jul. 2008.

RYAN, D.; MILIS, L.; MISRI, N. Depression During Pregnancy. Cam. Fam. Physician, 51, 1087-1093. 2005.

SABROZA, A. R., LEAL, M. C., SOUZA JR., P. R., \& GAMA, S. G. N. Algumas Repercussões Emocionais Negativas da Gravidez Precoce em Adolescentes do Município do Rio de Janeiro (1999-2001). Cadernos de Saúde Pública, 20(Sup. 1), p:130-37. 2004.

SANTOS, K. O. B., ARAÚJO, T. M. D., OLIVEIRA, N. F. D. Estrutura Fatorial e Consistência Interna do Self-Reporting Questionnaire (SRQ-20) em População Urbana. Cad. Saúde Pública, Rio de Janeiro, 25(1): 214-222, jan. 2009.

SCHUIMID, E; SCHIMID, L. P. C. A Incidência da Gravidez no Contexto da Adolescência Contemporânea. Rev. Med. Minas Gerais. 22(3): 328-333, 2012.

SILVA A. P. F., HIRAI N. K., SILVA M. E. Os Fatores Emocionais Gerados pela Gravidez na Adolescência. Conscientia e Saúde, 8(1):91-97. 2009.

SOUSA M. C. R., GOMES K. R. O. Conhecimento objetivo e percebido sobre contraceptivos hormonais orais entre adolescentes com antecedentes gestacionais. Cad. Saúde Pública. 25(3):645-54. 2009.

UFJF, Universidade Federal de Juiz de Fora. Laboratório de Demografia e Estudos Populacionais. Pobreza e abusos estimulam casamentos infantis no Brasil. set. 2015. Disponível em: <http://www.ufjf.br/ladem/2015/09/16/pobreza-e-abusos-estimulamcasamentos-infantis-no-brasil/> Acesso em: 28 set. 2015. 
VIEIRA M. L. F., BICALHO G. G., SILVA J. L. C.P., BARROS FILHO A. A. Crescimento e desenvolvimento de filhos de mães adolescentes no primeiro ano de vida. Rev Paul Pediatr. 25(4):343-8. 2007. 